\title{
Über die Autoren/Abstracts
}

\section{Peter Duesberg}

Geb. 1966 in Köln; arbeitete nach der germanistischen Promotion und Tätigkeiten als Lektor für Deutsch an Universitäten in Süd-Korea und Frankreich als Dozent für DaF in der kommunalen und kirchlichen Erwachsenenbildung sowie als Lehrbeauftragter im DaF-Bereich an der Heinrich-Heine Universität Düsseldorf.

\section{Axel Harting}

Lektor für Deutsch als Fremdsprache im fremdsprachendidaktischen Zentrum der Universität Hiroshima. Als Absolvent der Fächer Deutsch als Fremdsprache und Anglistik bis Anfang 2003 wissenschaftlicher Mitarbeiter im Bereich der studienbegleitenden Sprach- und Schreibförderung für internationale Studierende an der Universität Bielefeld. Forschungsschwerpunkte: Erwerb und Vermittlung fremdsprachlicher Schreibkompetenz, kontrastive Beschreibung kultureller Ausdrucksformen im Deutschen und Japanischen.

\section{Ingrid Laurien}

Geb. 1950, Dr. phil.; Studium der Germanistik, Geschichte und Politik, Promotion an der Universität Göttingen, Zweites Staatsexamen, 1981-1985 wissenschaftliche Mitarbeiterin am Historischen Seminar der Universität Göttingen. 1986-1992 Lektorin des DAAD an der Universität Nairobi, Kenia. Bis Ende 2004 Leitung des DAAD Informationsbüros Südafrika und Lektorin an der Deutschabteilung der University of the Witwatersrand, Johannesburg, Südafrika. Z.Zt. Arbeit an einem Projekt zur Landeskunde im Bereich Fremdsprachendidaktik an der Universität Potsdam.

\section{Tristan Lay}

Dr. phil., geb. 1978; Studium von Sprachlehrforschung und Sinologie in Bochum, Magister Artium 2002, 2004 Promotion am Seminar für Sprachlehrforschung an der Ruhr-Universität Bochum. Seit 2006 Assistant Professor für Deutsch als Fremdsprache am Europäischen Institut für Fremdsprachliche Philologien der National Chengchi University in Taipei. Forschungsschwerpunkte und Publikationen in den Bereichen Sprachlehrforschung, empirische Fremdsprachenerwerbsforschung, Fremdsprachendidaktik, insbesondere Didaktik und Methodik des Unterrichts Deutsch als Fremdsprache sowie Forschungen im Bereich des multiplen Sprachenlernens.

\section{Jörg Matthias Roche}

Professor für Deutsch als Fremdsprache; Leiter des Multimedia Forschungs- und Entwicklungslabors (»Werk-Stadt«) im Departement für Kommunikation und Sprachen an der Ludwig-MaximiliansUniversität München. Forschungsschwerpunkte: Theorie und Medien der Sprach- und Kulturvermittlung. Zahlreiche Veröffentlichungen; weitere Informationen, Projektbeschreibungen und Demos finden sich unter http://werkstadt.daf.uni-muenchen.de, www.linguaconsult.net und www.sprachchancen.de, www.uni-deutsch.de. Mitglied u. a. im Beirat Germanistik des Deutschen Akademischen Austauschdienstes, im Beirat Sprache des Goethe Institutes und den Wissenschaftlichen Beiräten der Zentralstelle für das Auslandsschulwesen und des FADAF, Mitglied des Wissenschaftlichen Beirates des Bundesamtes für Migration und Flüchtlinge (BAMF). 


\section{Torsten Schlak}

Dr. phil.; Studium der Fächer Deutsch als Fremdsprache, Sprachlehrforschung und Second Language Acquisition in Bielefeld, Bochum und Hawai'i. M. A. (1996); Dr. phil. (1999). 2000-2003 Hochschullehrer für Deutsch als Fremdsprache und Sprachlehrforschung an der Universität Osaka. Seit 2003 Juniorprofessor am Seminar für Sprachlehrforschung der RuhrUniversität Bochum.

\section{Thomas Stahl}

M. A.; Studium der Germanistik, Geschichte und Erziehungswissenschaften.
Zusatzausbildung >Deutsch als Fremdsprachenphilologie (Universität Regensburg). Aufbaustudiengang Japanische Sprache und Kultur< (Universität Tübingen). 2001-2003 WHK am Institut für Germanistik der Universität Regensburg, Tätigkeiten im Bereich DaF an Universitäten und anderen Bildungseinrichtungen in Deutschland und Japan. Seit 2003 DAADLektor an der Westböhmischen Universität Plzeň, Tschechische Republik. Lehraufträge an der Universität Regensburg. Veröffentlichungen zur deutschen und japanischen Literatur sowie im Bereich der Literatur- und Fremdsprachendidaktik.

\begin{tabular}{|c|c|c|c|c|c|}
\hline 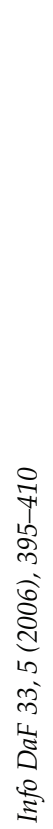 & 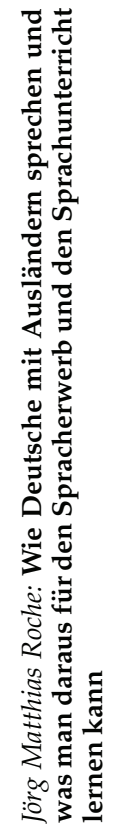 & 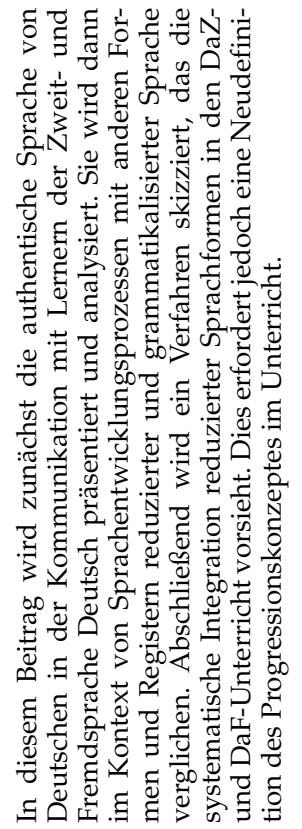 & 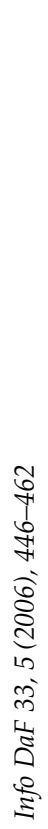 & 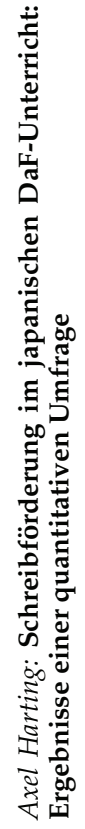 & 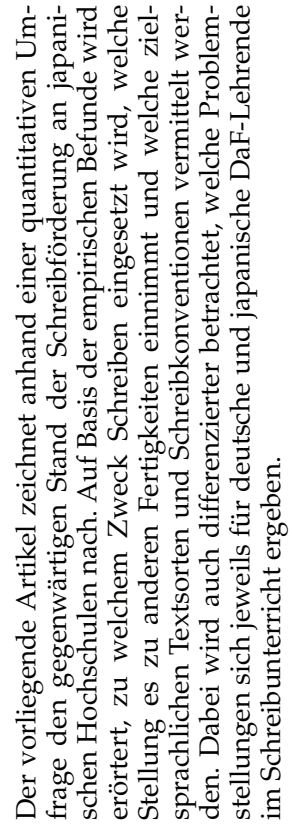 \\
\hline
\end{tabular}




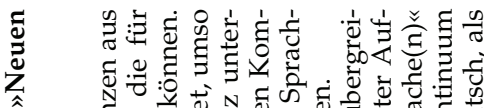

خ्रे N

.

ฮ ष्ष

踏

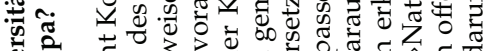

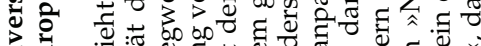

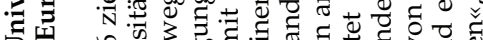

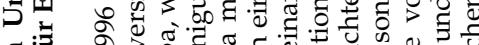

ส

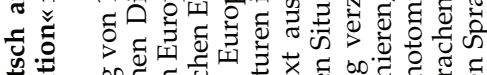

D. $0.0 \%$ च

ड़

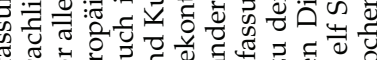

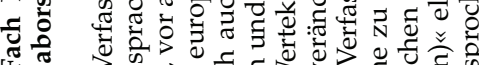

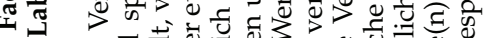

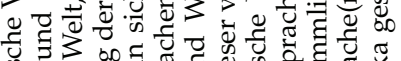

ค็

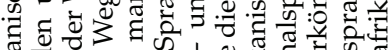

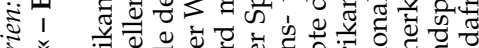

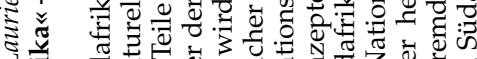

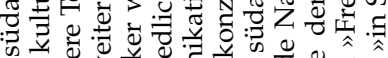

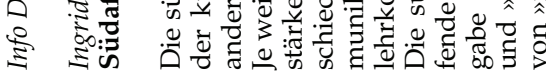

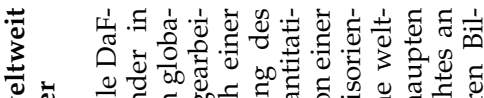

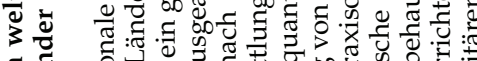

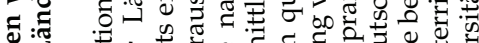

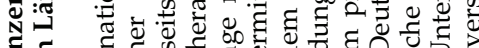

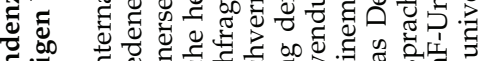

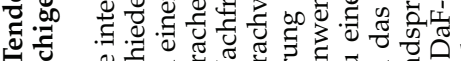

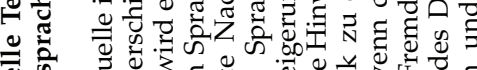

की

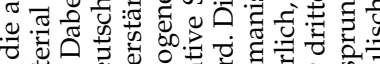

สं

人ิ هั ब छ

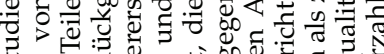
के 0 记

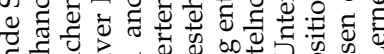

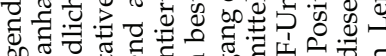

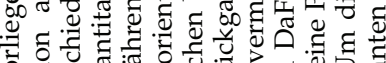

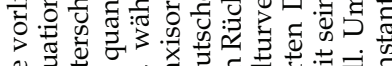

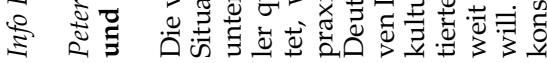

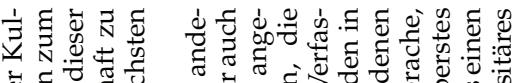

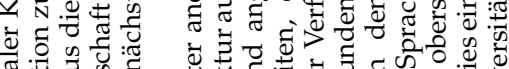

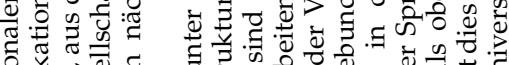

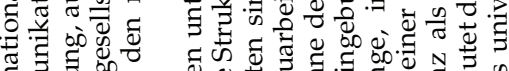

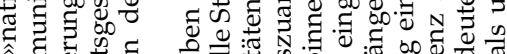

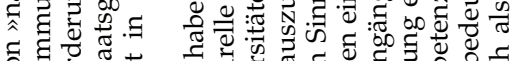

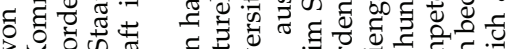
证 क

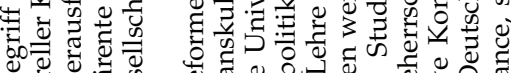

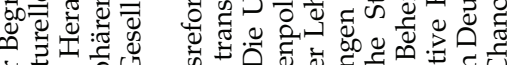

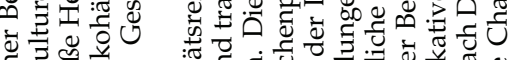

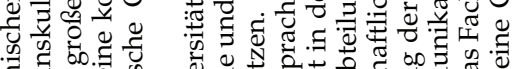
告

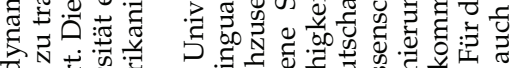

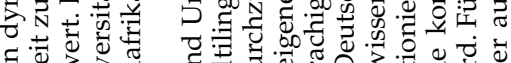

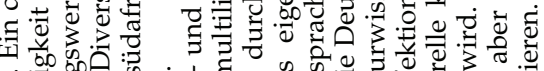

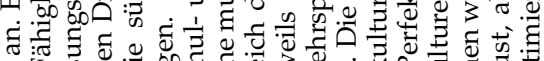

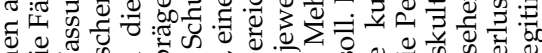

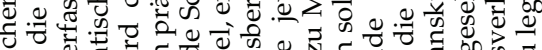

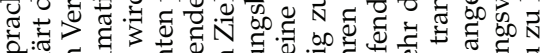

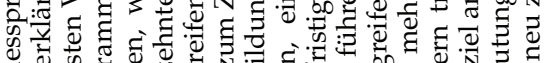

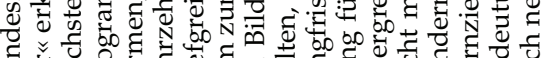

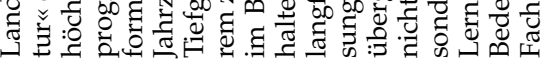

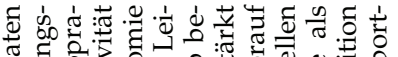
की a뭉 ธक्ष

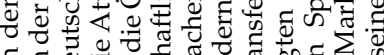

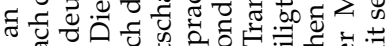
चृ दुष

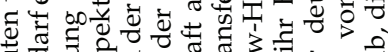

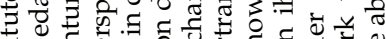
要航 돌 चै 范 S

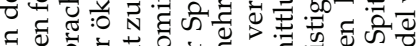
व

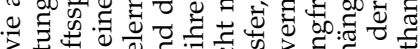
s. o. o

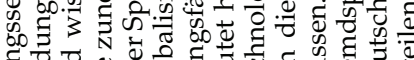

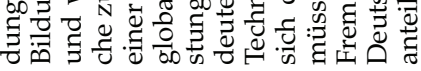




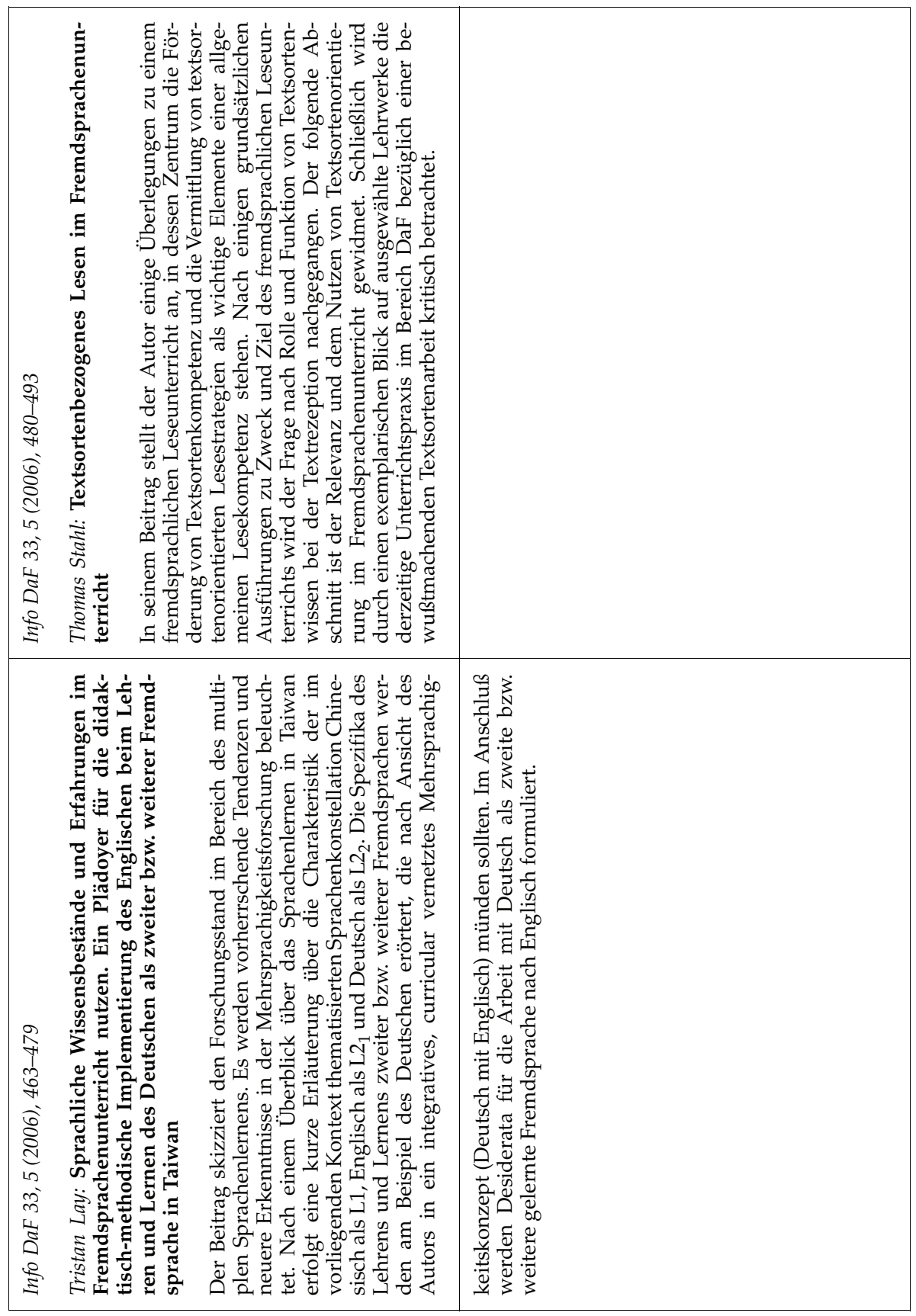


NEU Deutsch als Fremdsprache für Fortgeschrittene

\section{Mittelpunkt bringt es auf den Punkt.}

- Im Mittelpunkt steht der Erfolg des Lerners

- Komplette Prüfungsvorbereitung auf die neuen Goethe-Zertifikate B2 und C1

- Konsequente Orientierung am Europäischen Referenzrahmen
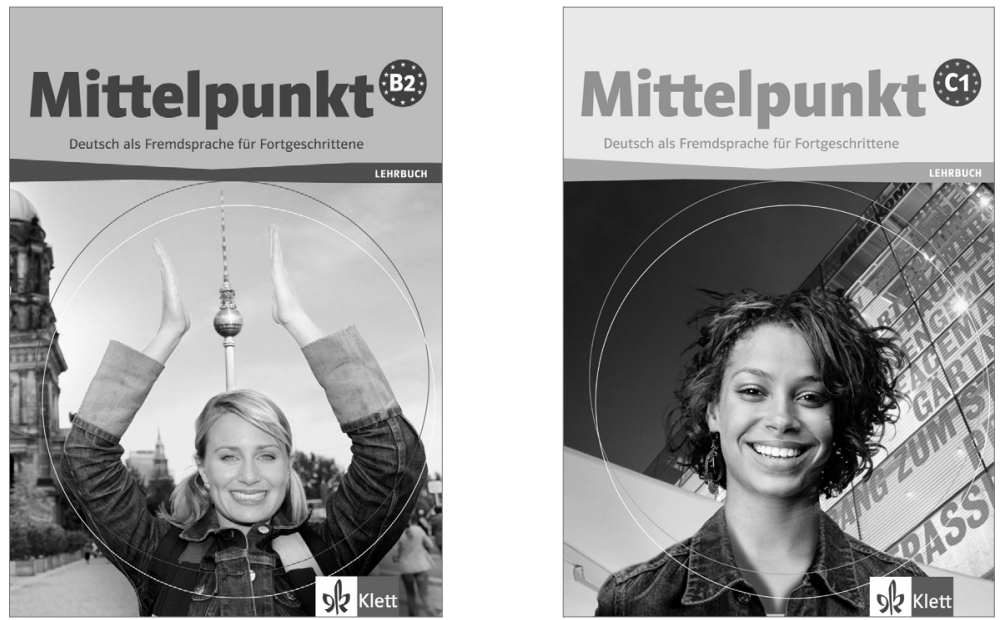

Mittelpunkt B2 erscheint im März 2007!

Bestellung und Beratung bei Klett:

Ernst Klett Sprachen, Postfach 102645, 70022 Stuttgart

Telefon $+49711 \cdot 6672-1010$, Telefax $+49711 \cdot 6672-2080$

www.klett-edition-deutsch.de 